\title{
Non-unitary leptonic mixing and leptogenesis
}

\author{
Stefan Antusch, ${ }^{a}$ Steve Blanchet, ${ }^{b}$ Mattias Blennow ${ }^{a}$ \\ and Enrique Fernandez-Martinez ${ }^{a}$ \\ ${ }^{a}$ Max-Planck-Institut für Physik (Werner-Heisenberg-Institut), \\ Föhringer Ring 6, 80805 München, Germany \\ ${ }^{b}$ Maryland Center for Fundamental Physics, University of Maryland, \\ College Park, MD, 20742, U.S.A. \\ E-mail: antusch@mppmu.mpg.de, sblanche@umd.edu, blennow@mppmu.mpg.de, \\ enfmarti@mppmu.mpg.de
}

ABSTRACT: We investigate the relation between non-unitarity of the leptonic mixing matrix and leptogenesis. We discuss how all parameters of the canonical type-I seesaw mechanism can, in principle, be reconstructed from the neutrino mass matrix and the deviation of the effective low-energy leptonic mixing matrix from unitary. When the mass $M^{\prime}$ of the lightest right-handed neutrino is much lighter than the masses of the others, we show that its decay asymmetries within flavour-dependent leptogenesis can be expressed in terms of two contributions, one depending on the unique dimension five $(d=5)$ operator generating neutrino masses and one depending on the dimension six $(d=6)$ operator associated with non-unitarity. In low-energy seesaw scenarios where small lepton number violation explains the smallness of neutrino masses, the lepton number conserving $d=6$ operator contribution generically dominates over the $d=5$ operator contribution which results in a strong enhancement of the flavour-dependent decay asymmetries without any resonance effects. To calculate the produced final baryon asymmetry, the flavour equilibration effects directly related to non-unitarity have to be taken into account. In a simple realization of this non-unitarity driven leptogenesis, the lower bound on $M^{\prime}$ is found to be about $10^{8} \mathrm{GeV}$ at the onset of the strong washout regime, more than one order of magnitude below the bound in "standard" thermal leptogenesis.

Keywords: Cosmology of Theories beyond the SM, Neutrino Physics, Beyond Standard Model, CP violation

ArXiv EPrint: 0910.5957 


\section{Contents}

1 Introduction 1

2 Non-unitarity relation to high-energy observables in the type-I seesaw model

3 The three-family low scale seesaw scenario 5

4 Leptogenesis in the three-family low-scale seesaw scenario $\quad 7$

$\begin{array}{lll}4.1 & \text { Flavour-dependent decay asymmetries } & 7\end{array}$

$\begin{array}{lll}4.2 & \text { Final baryon asymmetry } & 8\end{array}$

$\begin{array}{llr}5 & \text { Flavour equilibration } & 9\end{array}$

6 Summary and discussion $\quad 12$

\section{Introduction}

Non-unitarity of the leptonic mixing matrix at low energies is a generic manifestation of new physics in the lepton sector often related to the mechanism responsible for the generation of neutrino masses. Non-unitarity appears whenever additional heavy particles mix with the light neutrinos or their charged lepton partners. After integrating the heavy states out of the theory, the $3 \times 3$ submatrix of the light neutrinos remains as an effective mixing matrix. This low-energy leptonic mixing matrix is, in general, not unitary.

One example where non-unitarity is predicted is the generic type-I seesaw mechanism [1-4], where the Standard Model (SM) is extended by (typically three) right-handed neutrinos. If the type-I seesaw mechanism operates at energies as high as the Grand Unification scale (GUT scale), then non-unitarity effects are tiny. However, if the seesaw mechanism is realized at low energies close to the electroweak scale, then non-unitarity is enhanced and can be observable. It may then provide important hints to the origin of neutrino masses. It is important to note that, in low-energy seesaw scenarios, the smallness of the neutrino masses is not explained by the largeness of the seesaw scale. However it can be explained in a technically natural way by a lepton number symmetry which is broken only by a small amount [5].

While the neutrino masses in the type-I seesaw mechanism are effectively described by the unique lepton number violating Weinberg operator of $d=5$, the non-unitarity of the leptonic mixing matrix is generated by the lepton number conserving $d=6$ operator contributing to the kinetic terms of the neutrinos [6, 7]. In low-energy seesaw scenarios with approximately conserved lepton number, the $d=6$ operator can cause significant effects, since it is not suppressed by the smallness of the neutrino masses. 
One attractive feature of the seesaw mechanism is that it can explain the observed baryon asymmetry of the Universe via the mechanism of leptogenesis [8] (for a recent review, see ref.[9]). If the seesaw mechanism, and thus also the mechanism of thermal leptogenesis, operates at high energies, the decay asymmetries for leptogenesis are typically dominated by the $d=5$ operator. Unfortunately the high-energy parameters which control leptogenesis cannot be fully reconstructed from the measurements at low energy, since combinations of the Yukawa couplings different from those in the $d=5$ operator also appear.

On the other hand, if the seesaw mechanism operates at lower energies, predicting an observable non-unitarity of the leptonic mixing matrix, one may in principle obtain enough information from low-energy measurements to reconstruct the full Lagrangian and, therefore, the parameters that control leptogenesis $[7,10,11]$.

The purpose of this paper is to clarify the relation between the high-energy parameters that control successful leptogenesis and their low-energy manifestations, i.e., neutrino masses and mixings and deviations from unitarity of the leptonic mixing matrix, making special emphasis on the latter. In section 2 we describe in detail the method and conditions under which the full high-energy Lagrangian can be reconstructed from the low-energy effects. In section 3 we introduce a "minimalistic" low-scale seesaw model with three right-handed neutrinos in which the smallness of neutrino masses is explained by an approximate lepton number symmetry. However, deviations from unitary mixing induced by the $d=6$ operator are not protected by the symmetry and can be sizeable, leading to effects that could, in principle, be tested in precision electroweak measurements. In section 4 we discuss how, in this model, leptogenesis could be driven by the $d=6$ operator that induces the deviation from unitary mixing via flavoured leptogenesis. In section 5 we point out that the same $d=6$ operator that drives flavoured leptogenesis can also lead to a flavour equilibration, which could wash out the generation of lepton number. We also discuss the conditions under which successful leptogenesis can occur. Finally, in section 6, we summarize and discuss our results.

\section{Non-unitarity relation to high-energy observables in the type-I seesaw model}

In this section we describe how the full Lagrangian of the type-I seesaw can, in principle, be reconstructed from the low-energy observations of neutrino masses and mixings, including deviations from unitary mixing. The conditions under which this reconstruction is possible were described in ref.[7], while a method to realize the reconstruction was outlined in ref.[10]. Here we present a new algorithm to perform the reconstruction through which the high-energy parameters can be derived more easily.

Let us consider the Lagrangian of the standard type-I seesaw model which consists of the one for the SM plus an extra piece containing the allowed couplings between the SM fields and additional gauge singlet fermions (i.e., right-handed neutrinos) $N_{\mathrm{R}}^{i}$ :

$$
\mathscr{L}=\mathscr{L}_{\mathrm{SM}}-\frac{1}{2} \overline{N_{\mathrm{R}}^{i}} M_{i j}^{N} N_{\mathrm{R}}^{c j}-\left(Y_{N}\right)_{i \alpha} \overline{N_{\mathrm{R}}^{i}} \widetilde{\phi}^{\dagger} \ell_{\mathrm{L}}^{\alpha}+\text { H.c. } .
$$


Here, $\phi$ denotes the SM Higgs field, which breaks the electroweak (EW) symmetry after acquiring its vacuum expectation value (vev) $v_{\mathrm{EW}}$, and we have used the definition $\tilde{\phi}=i \tau_{2} \phi^{*}$.

The low-energy effects of the three-family low-scale seesaw model, from the point of view of neutrino oscillation experiments, is given by two effective operators, one of mass dimension five and one of mass dimension six. The $d=5$ operator is the ubiquitous lepton number violating Weinberg operator

$$
\delta \mathcal{L}^{d=5}=\frac{1}{2} c_{\alpha \beta}^{d=5}\left(\overline{L^{c}}{ }_{\alpha} \tilde{\phi}^{*}\right)\left(\tilde{\phi}^{\dagger} L_{\beta}\right)+\text { H.c. },
$$

which is the lowest-dimensional effective operator for generating neutrino masses using the field content of the SM. The coefficient matrix $c_{\alpha \beta}^{d=5}$ is

$$
c_{\alpha \beta}^{d=5}=-\left(Y_{N}^{T}\right)_{\alpha i}\left(M_{N}\right)_{i j}^{-1}\left(Y_{N}\right)_{j \beta}
$$

and relates to the low-energy neutrino mass matrix as

$$
m_{\nu}=v_{\mathrm{EW}}^{2} c^{d=5}
$$

The effective $d=6$ operator

$$
\delta \mathcal{L}^{d=6}=c_{\alpha \beta}^{d=6}\left(\bar{L}_{\alpha} \tilde{\phi}\right) i \not \partial\left(\tilde{\phi}^{\dagger} L_{\beta}\right)
$$

conserves lepton number and, after EW symmetry breaking, contributes to the kinetic terms of the neutrinos. After their canonical normalization, they generate a non-unitary leptonic mixing matrix $N$ as well as non-universal couplings proportional to $N^{\dagger} N$ of the neutrinos to the $Z$ boson (see, e.g., refs. $[6,7,12]$ ). The coefficient matrix $c_{\alpha \beta}^{d=6}$ is given by (see, e.g., ref.[7])

$$
c_{\alpha \beta}^{d=6}=\sum_{i}\left(Y_{N}^{\dagger}\right)_{\alpha i}\left(M_{N}\right)_{i i}^{-2}\left(Y_{N}\right)_{i \beta},
$$

in the basis where $M_{N}$ is diagonal.

If we parametrize the non-unitary leptonic mixing matrix $N$ as [13]

$$
N=(1+\eta) U
$$

where $\eta$ is Hermitian and $U$ is unitary, then $\eta_{\alpha \beta}$ is related to the coefficient matrix $c_{\alpha \beta}^{d=6}$ by

$$
\eta_{\alpha \beta}=-v_{\mathrm{EW}}^{2} c_{\alpha \beta}^{d=6} / 2
$$

In refs. [12, 14-17], the following constraints on these parameters at the $90 \%$ C.L. were derived: $\eta_{e e}<2.0 \cdot 10^{-3}, \eta_{e \mu}<5.9 \cdot 10^{-5}, \eta_{e \tau}<1.6 \cdot 10^{-3}, \eta_{\mu \mu}<8.2 \cdot 10^{-4}, \eta_{\mu \tau}<1.0 \cdot 10^{-3}$ and $\eta_{\tau \tau}<2.6 \cdot 10^{-3}$.

In the type-I seesaw the full $6 \times 6$ mixing matrix $U_{\text {tot }}$ is the unitary matrix that diagonalizes the extended neutrino mass matrix:

$$
U_{\text {tot }}^{T}\left(\begin{array}{cc}
0 & m_{D}^{T} \\
m_{D} & M_{N}
\end{array}\right) U_{\text {tot }}=\left(\begin{array}{cc}
m & 0 \\
0 & M
\end{array}\right),
$$


where $m_{D}=v_{\mathrm{EW}} Y_{N}$ and $M_{N}$ are the neutrino's Dirac and Majorana mass matrices and $m$ and $M$ are diagonal matrices. It is easier to perform the diagonalization in two steps: first a block-diagonalization and then two unitary rotations to diagonalize the mass matrices of the light and heavy neutrinos, i.e.,

$$
U_{\mathrm{tot}}=\left(\begin{array}{cc}
A & B \\
C & D
\end{array}\right)\left(\begin{array}{cc}
U & 0 \\
0 & V
\end{array}\right)
$$

where $U$ and $V$ are unitary matrices. Without loss of generality, we can choose a basis for the heavy singlets such that $V=I$ and $M_{N}=M$. When performing the block diagonalization, the mixing between the light and heavy neutrinos is suppressed when $M_{N}>m_{D}$ so that

$$
B \simeq \Theta=m_{D}^{\dagger} M_{N}^{-1} .
$$

We can exploit the suppression of eq. (2.11) to write the unitary block diagonalization as the exponential expansion of an anti-Hermitian matrix:

$$
\left(\begin{array}{ll}
A & B \\
C & D
\end{array}\right)=\exp \left(\begin{array}{cc}
0 & \Theta \\
-\Theta^{\dagger} & 0
\end{array}\right)=\left(\begin{array}{cc}
1-\frac{1}{2} \Theta^{\dagger} & \Theta \\
-\Theta^{\dagger} & 1-\frac{1}{2} \Theta^{\dagger} \Theta
\end{array}\right)+\mathcal{O}\left(\Theta^{3}\right) .
$$

The block-diagonalization yields the complex symmetric neutrino mass matrix

$$
m_{\nu}=-m_{D}^{T} M_{N}^{-1} m_{D}
$$

which can be diagonalized by a unitary transformation $U$ such that $m=\operatorname{diag}\left(m_{1}, m_{2}, m_{3}\right)=$ $U^{T} m_{\nu} U$. Notice that the mixing matrix of the three light neutrinos is given by

$$
N=A U=(1+\eta) U=\left(1-\Theta \Theta^{\dagger} / 2\right) U,
$$

As described above, $\eta=-\Theta \Theta^{\dagger} / 2$ exactly contains the coefficients $c^{d=6}$ of the $d=6$ operator. In particular, note that this implies that $c^{d=6}$ is Hermitian and positive semidefinite.

Assuming that the low-energy observables $U, m$ and $\eta$ have been measured, it is then natural to ask the question of whether one can reconstruct the high-energy parameters contained in $m_{D}$ and $M$. In order to split the low- and high-energy observables, we make use of the parametrization proposed in ref.[18] for the Dirac mass matrix $m_{D}$, which is introduced as follows: From eq. (2.13) we have that $-\sqrt{m^{-1}} U^{T} m_{D}^{T} M^{-1} m_{D} U \sqrt{m^{-1}}=1$. Thus, defining $R \equiv i \sqrt{M^{-1}} m_{D} U \sqrt{m^{-1}}$, we have the condition $R^{T} R=1$. We will consider here the case in which the number of heavy right-handed singlets is equal to the number of light neutrinos, and thus, the matrix $R$ must be a complex orthogonal matrix. Multiplying by inverses of matrices, the definition of $R$ can be rewritten as

$$
m_{D}=-i \sqrt{M} R \sqrt{m} U^{\dagger}
$$

Notice that the matrix $U$ that appears in the parametrization of eq. (2.15) is not the neutrino mixing matrix that describes the neutrino couplings in charged-current (CC) interactions $N=(1+\eta) U$, but only the unitary part of the CC mixing diagonalizing $m_{\nu}$. 
In eq. (2.15) the observables of the $d=5$ operator describing the light neutrino mass matrix are all contained within $U$ and $m$, while $R$ and $M$ contain the missing information in order to reconstruct the high-energy parameters. In order to perform the reconstruction we will assume that both the $d=5$ operator, i.e., $U$ and $m$, and the $d=6$ operator $\eta$ are known. Notice that the deviations from unitary mixing encoded in $\eta$ can be probed and potentially measured through electroweak decays $[12,14-16,19]$ as well as neutrino oscillation experiments [13, 20-24].

Using the parametrization of eq. (2.15) in the expression for the $d=6$ operator $2 \eta=$ $-m_{D}^{\dagger} M^{-2} m_{D}$, we define the matrix $H$ as

$$
H \equiv-\sqrt{m^{-1}} U^{\dagger} 2 \eta U \sqrt{m^{-1}}=\left(R^{*}\right)^{-1} M^{-1} R .
$$

With this definition, $H$ is a positive semidefinite Hermitian matrix, since it can be decomposed as $F F^{\dagger}$, and contains all of the available low-energy information. Equation (2.16) is known as the conjugate diagonalization (or simply the "condiagonalization") of the matrix $H$. Notice that replacing $\left(R^{*}\right)^{-1}$ by $R^{-1}$ in eq. (2.16) would reduce it to a normal diagonalization. It can be shown that all Hermitian positive definite matrices can be condiagonalized and the solution is unique under the requirement that the $M_{i}$ are real and positive. The simplest way of reconstructing $R$ and $M$ from the matrix $H$ is to note that $H^{*} H=H^{T} H$ is a complex symmetric matrix and that

$$
H^{*} H=R^{-1} M^{-1} R^{*}\left(R^{*}\right)^{-1} M^{-1} R=R^{T} M^{-2} R,
$$

and thus, $R$ is the complex orthogonal matrix which diagonalizes $H^{*} H$ with corresponding eigenvalues $M_{i}^{-2}$. It should be noted that the reconstruction of $R$ and $M$ has been previously studied in ref.[10]. However, the simple reconstruction algorithm presented here, only involving the diagonalization of a complex symmetric matrix, is new. Notice that, for the algorithm to work and for eq. (2.16) to have solutions, it is vital that the matrix $H$ is a positive definite matrix. This will be guaranteed as long as the $d=6$ operator $c^{d=6}$ is also positive definite. By construction $c^{d=6}$ is positive semidefinite so the only case in which $H$ is not positive definite is when $c^{d=6}$ has one or more zero eigenvalues. This could happen for two reasons: either the number of heavy right-handed neutrinos is smaller than the number of light neutrinos, or the rows of the Yukawa matrix are not linearly independent. In any of these cases the number of parameters in the high-energy theory is smaller than in the full case considered here and the reconstruction of the Lagrangian would then be easier, sometimes without the need of involving the $d=6$ operator but only through $U$ and $m$ (see, e.g., ref.[25]). If, on the other hand, the number of heavy right-handed singlets is larger than the number of light neutrinos, then the information encoded in the $d=6$ operator $\eta$ plus the neutrino masses and mixings $m$ and $U$ is not sufficient to reconstruct the full high-energy Lagrangian. We find these limitations to be in agreement with ref.[10], where the reconstruction algorithm relies on $\eta$ being invertible.

\section{The three-family low scale seesaw scenario}

The "minimalistic" low-scale seesaw model we present here is a type-I seesaw model with three right-handed neutrinos to which we additionally impose a softly broken "lepton 
number"-like (global) U(1) symmetry where the charge of the $\mathrm{SU}(2)_{\mathrm{L}}$ doublets $L_{\alpha}$ is opposite to that of the field $N_{\mathrm{R}}^{3}$ but equal to that of the field $N_{\mathrm{R}}^{2}$. We assign zero lepton number to $N_{\mathrm{R}}^{1}$. In the symmetry limit, $M_{i j}^{N}$ and $Y_{N}$ are forced to have the form:

$$
Y_{N}=\left(\begin{array}{ccc}
0 & 0 & 0 \\
y_{e} & y_{\mu} & y_{\tau} \\
0 & 0 & 0
\end{array}\right), \quad M^{N}=\left(\begin{array}{ccc}
M^{\prime} & 0 & 0 \\
0 & 0 & M \\
0 & M & 0
\end{array}\right) \text {. }
$$

At this level, the neutrinos are exactly massless, but non-unitarity of the leptonic mixing matrix is already induced. When small soft breaking terms $\mu_{i}$ and $\mu_{i}^{\prime}$ are allowed, this rigid structure is perturbed to

$$
Y_{N}=\left(\begin{array}{ccc}
\mu_{e}^{\prime} & \mu_{\mu}^{\prime} & \mu_{\tau}^{\prime} \\
y_{e} & y_{\mu} & y_{\tau} \\
\mu_{e} & \mu_{\mu} & \mu_{\tau}
\end{array}\right), \quad M^{N}=\left(\begin{array}{ccc}
M^{\prime} & \mu_{4}^{\prime} & \mu_{5}^{\prime} \\
\mu_{4}^{\prime} & \mu_{4} & M \\
\mu_{5}^{\prime} & M & \mu_{5}
\end{array}\right),
$$

and masses for the light neutrinos, suppressed by the small $\mu_{i}$ and $\mu_{i}^{\prime}$ parameters, are generated. Notice that the $d=6$ operator is not protected by the lepton number symmetry and would have a large leading order contribution

$$
c_{\alpha \beta}^{d=6}=\frac{y_{\alpha}^{*} y_{\beta}}{M^{2}}+\ldots,
$$

where the dots denote sub-leading terms proportional to $\mu_{\alpha}$ (or $\mu_{4} / M, \mu_{5} / M$ ) and $\mu_{\alpha}^{\prime}$ (or $\left.\mu_{4}^{\prime} / M, \mu_{5}^{\prime} / M\right)$. In particular, the six moduli $\left|\eta_{\alpha \beta}\right|$ depend only on the three parameters $\left|y_{e} / M\right|,\left|y_{\mu} / M\right|,\left|y_{\tau} / M\right|$. This implies that at leading order the rank of the $d=6$ operator is one and that the reconstruction algorithm presented in section 2 would not be applicable. On the other hand, the number of parameters contributing to the $d=5$ and $d=6$ operator at leading order in this model is very limited and they can actually be reconstructed with information mainly from $m$ and $U$. Indeed, the $d=5$ operator is given by [25]:

$$
c_{\alpha \beta}^{d=5}=\left(\mu_{\alpha}-\frac{\mu_{5}}{M} y_{\alpha}\right) \frac{1}{M} y_{\beta}+y_{\alpha} \frac{1}{M}\left(\mu_{\beta}-\frac{\mu_{5}}{M} y_{\beta}\right)+\ldots .
$$

Following ref.[25] both vectors $y_{\alpha}$ and $\mu_{\alpha}-\left(\mu_{5} / M\right) y_{\alpha}$ can be reconstructed from $m$ and $U$ up to an overall normalization. In particular,

$$
\begin{aligned}
y_{\alpha} & \propto \sqrt{1+\rho} U_{\alpha 3}^{*}+\sqrt{1-\rho} U_{\alpha 2}^{*}, \\
\rho & =\frac{\sqrt{1+r}-\sqrt{r}}{\sqrt{1+r}+\sqrt{r}}
\end{aligned}
$$

for normal hierarchy and

$$
\begin{aligned}
y_{\alpha} & \propto \sqrt{1+\rho} U_{\alpha 2}^{*}+\sqrt{1-\rho} U_{\alpha 1}^{*}, \\
\rho & =\frac{\sqrt{1+r}-1}{\sqrt{1+r}+1}
\end{aligned}
$$

for inverted hierarchy. Here, $r=\left|\Delta m_{21}^{2}\right| /\left|\Delta m_{31}^{2}\right|$. If terms beyond leading order are considered for the $d=6$ operator, then the reconstruction described in section 2 can be applied. In principle, this would also allow the extraction of the first Yukawa row $Y_{\alpha 1}=\mu_{\alpha}^{\prime}$ if the $d=6$ operator is known with sufficient precision. 


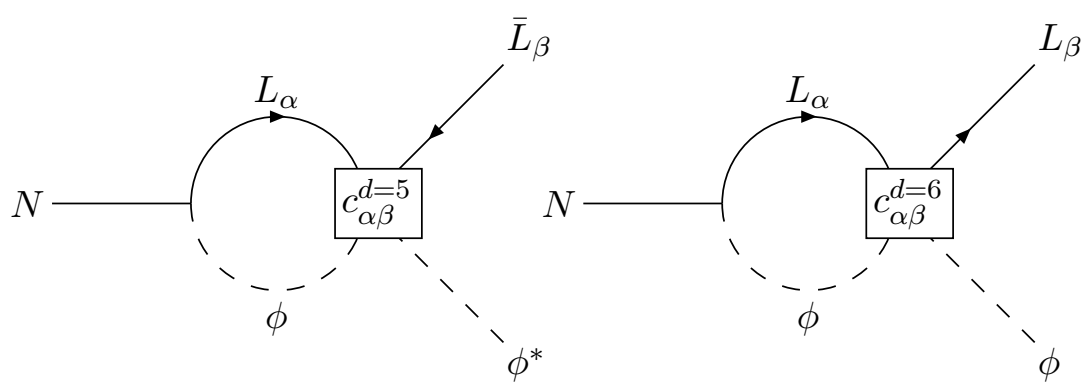

Figure 1. Effective operator decomposition of the diagrams leading to leptogenesis.

\section{Leptogenesis in the three-family low-scale seesaw scenario}

\subsection{Flavour-dependent decay asymmetries}

The flavour-dependent $C P$ asymmetries for the lightest right-handed neutrino (with mass $\left.M^{\prime}\right)$ in the lepton flavour $\alpha$ are given by

$$
\varepsilon_{1, \alpha} \simeq \frac{1}{8 \pi\left(Y Y^{\dagger}\right)_{11}} \sum_{j \neq 1}\left\{\operatorname{Im}\left[Y_{\alpha 1}^{T} Y_{\alpha j}^{\dagger}\left(Y Y^{\dagger}\right)_{1 j} \frac{3}{2 \sqrt{x_{j}}}+Y_{\alpha 1}^{T} Y_{\alpha j}^{\dagger}\left(Y Y^{\dagger}\right)_{j 1} \frac{1}{x_{j}}\right]\right\}
$$

where $x_{j}=M_{j}^{2} / M_{1}^{2} \gg 1$ was assumed (with $j=2,3, M_{1} \equiv M^{\prime}$, and with $M_{2}, M_{3}$ being the masses of $N_{\mathrm{R}}^{2}$ and $N_{\mathrm{R}}^{3}$ ). It is then possible to rewrite $\varepsilon_{1, \alpha}$ as the sum of two contributions, that of the $d=5$ operator and that of the $d=6$ operator (see figure 1 ):

$$
\begin{aligned}
\varepsilon_{1, \alpha} & \simeq \frac{1}{8 \pi\left(Y^{\dagger}\right)_{11}} \sum_{\beta}\left\{\operatorname{Im}\left[-\frac{3 M^{\prime}}{2} Y_{1 \alpha}^{*} c_{\alpha \beta}^{d=5} Y_{\beta 1}^{\dagger}+M^{\prime 2} Y_{1 \alpha} c_{\alpha \beta}^{d=6} Y_{\beta 1}^{\dagger}\right]\right\} \\
& \simeq \frac{M^{\prime 2}}{8 \pi\left(Y Y^{\dagger}\right)_{11}} \sum_{\beta}\left\{\operatorname{Im}\left[Y_{1 \alpha} c_{\alpha \beta}^{d=6} Y_{\beta 1}^{\dagger}\right]\right\},
\end{aligned}
$$

where, in the last step, we have neglected the contribution from the $d=5$ operator, since it is protected by the lepton number symmetry that explains the smallness of neutrino masses, which is not the case for the $d=6$ operator. Assuming the presence of an order one phase contribution to the imaginary part in eq. (4.2), as well as $\left|Y_{1 e}\right| \sim\left|Y_{1 \mu}\right| \sim\left|Y_{1 \tau}\right|$, we can further simplify this expression and express it in terms of the non-unitarity parameters in eq. (2.8):

$$
\varepsilon_{1, \alpha} \simeq \frac{M^{\prime 2}}{24 \pi} \sum_{\beta \neq \alpha} c_{\alpha \beta}^{d=6}=-\frac{1}{12 \pi} \frac{M^{\prime 2}}{v_{\mathrm{EW}}^{2}} \sum_{\beta \neq \alpha} \eta_{\alpha \beta} .
$$

For $M^{\prime}=1 \mathrm{TeV}$ and $\eta_{\alpha \beta}$ of order $10^{-4}$ we obtain a large $C P$ asymmetry $\varepsilon_{1, \alpha}$ of order $10^{-3}$ without resorting to the usual enhancement for quasi-degenerate masses of the heavy neutrinos $[26,27]$. As the contribution to $\varepsilon_{1, \alpha}$ originates from the $d=6$ operator (see also ref.[28]), we will refer to this scenario as non-unitarity driven leptogenesis.

It is crucial for our scenario that leptogenesis occurs when flavour effects are relevant $[29,30]$, i.e., for a right-handed neutrino mass $M^{\prime}<10^{12} \mathrm{GeV}$, so that the flavoured 
$C P$ asymmetries need to be considered instead of the total one $\varepsilon_{1}=\sum_{\alpha} \varepsilon_{1, \alpha}$, which is suppressed by the neutrino mass operator $(d=5)$. As we will see next, another requirement for a non-zero baryon asymmetry is that the washout in each flavour is different. This is therefore an example of purely flavoured leptogenesis [30, 31], where the asymmetry is generated exclusively due to flavour effects.

\subsection{Final baryon asymmetry}

An important parameter for flavoured leptogenesis is given by the decay (or washout) parameter, $K_{i \alpha}$, induced by the $\mathrm{RH}$ neutrino $N_{i}$, defined as the decay width over the Hubble expansion rate when $T=M_{i}$ :

$$
K_{i \alpha} \equiv \frac{\Gamma_{D}\left(N_{i} \rightarrow \ell_{\alpha} \phi+\overline{\ell_{\alpha}} \phi^{\dagger}\right)}{H\left(T=M_{i}\right)}=\frac{\left|Y_{i \alpha}\right|^{2} v_{\mathrm{EW}}^{2}}{m_{\star} M_{i}}
$$

where $m_{\star} \simeq 1.08 \times 10^{-3} \mathrm{eV}$ [32]. For future use, we also define $K_{i} \equiv \sum_{\alpha} K_{i \alpha}$.

With the assumption $M^{\prime} \ll M$ and $M^{\prime}<10^{9} \mathrm{GeV}$, the asymmetry is generated by $N_{1}$ in the three-flavour regime $[29,30]$. The contributions from the heavier states are exponentially washed out in all flavours, as long as $K_{1 \alpha} \gtrsim 3, \forall \alpha \in\{e, \mu, \tau\}$ [33]. Moreover, as pointed out above, since the $C P$ asymmetry is non-zero due to a pure flavour effect, it is crucial for the generation of the asymmetry that the washout is different in each flavour. Therefore, for simplicity, we will assume that the asymmetry is dominantly generated in one flavour, i.e., $\eta_{B \alpha} \simeq \eta_{B}$. The flavoured final asymmetry can be expressed as

$$
\eta_{B \alpha}=0.88 \times 10^{-2} \varepsilon_{1, \alpha} \kappa\left(K_{1 \alpha}\right)
$$

where it was assumed that sphalerons decouple after the electroweak phase transition [34, 35 ]. For $K_{1 \alpha} \gtrsim 3$, the efficiency factor $\kappa$ can be approximated as [36]

$$
\kappa\left(K_{1 \alpha}\right) \simeq \frac{0.5}{K_{1 \alpha}^{1.16}}
$$

In order to focus on the more relevant non-unitarity parameters and the mass scale $M^{\prime}$, we will for now fix a washout $K_{1 \alpha}=5$, which is a typical value in the strong washout regime. This requires Yukawa couplings $\left|Y_{1 \alpha}\right| \sim 10^{-6}$, as needed for the TeV-scale seesaw mechanism. We further assume the value $K_{1} \equiv \sum_{\gamma} K_{1 \gamma}=45$, such that $K_{1 \alpha} \ll K_{1 \beta \neq \alpha}$. In other words, in terms of Yukawa couplings, we have the relation $2\left|Y_{1 \alpha}\right|=\left|Y_{1 \beta \neq \alpha}\right|$. Using eqs. (4.2), (4.5) and (4.6), we have

$$
\eta_{B \alpha} \simeq-\frac{0.88 \times 10^{-2}}{36 \pi 5^{1.16}} \frac{M^{\prime 2}}{v_{\mathrm{EW}}^{2}} \sum_{\beta \neq \alpha} \eta_{\alpha \beta} \simeq 0.6 \times 10^{-5}\left(\frac{M^{\prime}}{M}\right)^{2} \sum_{\beta \neq \alpha} y_{\alpha}^{*} y_{\beta} .
$$

This prediction should be compared to the measured value $\eta_{B}^{\mathrm{CMB}}=(6.2 \pm 0.15) \times 10^{-10}[37]$. With Yukawa couplings $y_{\beta} \sim \mathcal{O}(1)$, it is easy to see that leptogenesis is possible with a mild hierarchy $M^{\prime} / M \sim 10^{-2}$. Moreover, it is interesting to see that the scale of leptogenesis can be lowered, at least in principle, to the weak scale. This is possible in our scenario because the purely flavoured contribution to the $C P$ asymmetry is not suppressed by the neutrino 
mass operator as in the usual case. The lower bound on the scale of leptogenesis [38-41], given by $M_{1}>3 \times 10^{9} \mathrm{GeV}$ at the onset of the strong washout, therefore does not apply. This was already noticed in ref.[33], where, using the Casas-Ibarra parametrization [18] in the limit $\left|\omega_{32}\right| \gg 1$, it was shown that the scale of leptogenesis could be lowered; the inverse seesaw model can be shown to correspond to the extreme case $\left|\omega_{32}\right| \gg 100$. The seesaw model under consideration could therefore potentially offer an alternative to quasidegenerate RH neutrinos [26, 27] to evade the gravitino bounds [42-45].

\section{Flavour equilibration}

From the previous discussion it seems that the scale of leptogenesis could be lowered to the weak scale without any problem. However, an important effect was neglected, namely flavour equilibration [46]. If flavours equilibrate, the final baryon asymmetry is proportional to the total $C P$ asymmetry $\varepsilon_{1}=\sum_{\alpha} \varepsilon_{1 \alpha}$, which is suppressed by the $d=5$ neutrino mass operator. We would thus recover the standard scenario, and the usual lower bound would apply.

Let us now estimate how efficient flavour equilibration is in our case. The main processes are $\Delta L=0$ scatterings with off-shell $N_{2}$ and $N_{3}$, e.g., $\ell_{\alpha} \phi \rightarrow \ell_{\beta} \phi$. Contrary to standard leptogenesis, the rates can be large, since the Yukawa couplings $y_{\alpha}$ are not constrained by neutrino masses, and therefore flavour equilibration is potentially a problem. The question is to what extent it reduces the available parameter space.

There are three different channels contributing to $\Delta L=0$ scatterings: $s$-channel $\ell_{\alpha} \phi \rightarrow \ell_{\beta} \phi, t$-channel $\ell_{\alpha} \phi^{\dagger} \rightarrow \ell_{\beta} \phi^{\dagger}$, and $t$-channel $\ell_{\alpha} \ell_{\beta}^{c} \rightarrow \phi \phi^{\dagger}$. The reduced cross-sections for these processes can be found in ref.[47], and in the limit $M^{\prime} \ll M$ the total $\Delta L=0$ cross-section is given by

$$
\hat{\sigma}_{\alpha \beta}(x) \simeq \frac{5}{4} \frac{\left|y_{\alpha}\right|^{2}\left|y_{\beta}\right|^{2}}{\pi}\left(\frac{M^{\prime}}{M}\right)^{2} x
$$

where $x \equiv s / M^{\prime 2}$. Note that the $N_{2}$ and $N_{3}$ contributions are essentially equal. The reaction rate is then obtained using

$$
\Gamma_{\alpha \beta}^{\Delta L=0} \equiv \frac{M^{\prime} z^{2}}{96 \pi^{2} \zeta(3)} \int_{x_{\mathrm{thr}}}^{\infty} d x \sqrt{x} \mathcal{K}_{1}(z \sqrt{x}) \hat{\sigma}(x),
$$

where $z \equiv M^{\prime} / T$ and $\mathcal{K}_{1}$ is the modified Bessel function of the second kind. This rate, as all the rates entering the Boltzmann equations, will be compared to the Hubble expansion rate, given by

$$
H(z)=1.66 \sqrt{g_{\star}} \frac{M^{\prime 2}}{z^{2} M_{\mathrm{Pl}}},
$$

where $g_{\star}=106.75$ and $M_{\mathrm{Pl}}=1.22 \times 10^{19} \mathrm{GeV}$.

The Boltzmann equations for leptogenesis are given by

$$
\begin{aligned}
& \frac{\mathrm{d} N_{N_{1}}}{\mathrm{~d} z}=-D\left(N_{N_{1}}-N_{N_{1}}^{\mathrm{eq}}\right), \\
& \frac{\mathrm{d} N_{\Delta_{\alpha}}}{\mathrm{d} z}=\varepsilon_{1 \alpha} D\left(N_{N_{1}}-N_{N_{1}}^{\mathrm{eq}}\right)-W_{\alpha}^{\mathrm{ID}} N_{\Delta_{\alpha}}-\sum_{\beta \neq \alpha} S_{\alpha \beta}^{\Delta L=0}\left(N_{\Delta_{\alpha}}-N_{\Delta_{\beta}}\right),
\end{aligned}
$$


where $N_{X}$ denotes any particle number or asymmetry $X$ in a portion of comoving volume containing one heavy neutrino in ultra-relativistic thermal equilibrium, so that $N_{N_{1}}^{\mathrm{eq}}(T \gg$ $\left.M_{1}\right)=1$. As a function of $z$, the equilibrium $\mathrm{RH}$ neutrino number density is given by $N_{N_{1}}^{\text {eq }}=0.5 z^{2} \mathcal{K}_{2}(z)$. Furthermore, we have defined $\Delta_{\alpha} \equiv \Delta B / 3-\Delta L_{\alpha}$. The normalized decay rate is given by $D \equiv \Gamma_{D} /[H(z) z]=K_{1} z\langle 1 / \gamma\rangle$ with the thermally averaged dilation factor $\langle 1 / \gamma\rangle$ given by the ratio of the modified Bessel functions $\mathcal{K}_{1}(z) / \mathcal{K}_{2}(z)$. Finally, we defined the normalized inverse decay rate $W_{\alpha}^{\mathrm{ID}} \equiv \Gamma_{\alpha}^{\mathrm{ID}} /[H(z) z]=0.25 K_{1 \alpha} \mathcal{K}_{1}(z) z^{3}$, and $S_{\alpha \beta}^{\Delta L=0} \equiv \Gamma_{\alpha \beta}^{\Delta L=0} /[H(z) z]$. Note that the normalized scattering rate is fitted within $10 \%$ by

$$
S_{\alpha \beta}^{\Delta L=0} \simeq 6.5 \times 10^{-4}\left|y_{\alpha}\right|^{2}\left|y_{\beta}\right|^{2}\left(\frac{M^{\prime} M_{\mathrm{Pl}}}{M^{2}}\right) z^{-2} .
$$

In the Boltzmann equations above, spectator processes $[48,49]$ and the conversion of a lepton flavour asymmetry into a $\Delta_{\alpha}$ asymmetry have been neglected, but we have checked that they do not change our results by more than $20 \%$. We have also checked that $\Delta L=1$ scatterings contribute subdominantly in the strong washout regime under consideration. As for $\Delta L=2$ processes their rates are suppressed by the small neutrino masses and can be safely neglected.

We show in figure 2 how the scattering rate normalized to the Hubble expansion rate varies with $M^{\prime}$, for a fixed hierarchy $M / M^{\prime}=10$ and washout $K_{1 \alpha}=3.5$, and for Yukawa couplings such that the baryon asymmetry of the Universe is produced [c.f. eq. (4.7)]. The shaded regions denote the asymmetry production time, roughly when $z_{B}-2<z<z_{B}+2$ with $z_{B}(K) \simeq 2+4 K^{0.13} \exp (-2.5 / K) \simeq 5$ [50]. Note that even though the flavour equilibrating scattering rate falls out of equilibrium before entering the shaded region for $M^{\prime} \gtrsim 3 \times 10^{8} \mathrm{GeV}$, there is still a residual effect, which suppresses the asymmetry by a factor of $2-3$. The reason is that the scattering rate is within a factor of 2 of the inverse decay rate during all the asymmetry production, and therefore some flavour equilibration is achieved.

Since $\Gamma^{\Delta L=0} / H \propto 1 / M^{\prime}$ for a fixed mass hierarchy $M^{\prime} / M$, it is clear that the scale of leptogenesis cannot be arbitrarily low. We have solved numerically the system of Boltzmann equations (5.4) and (5.5) without neglecting the contribution from any flavour, so that

$$
\eta_{B}=0.88 \times 10^{-2} \sum_{\alpha} \varepsilon_{1, \alpha} \kappa\left(K_{1 \alpha}\right)
$$

and we have required that the final asymmetry should fall within the $3 \sigma$ range of the observed baryon asymmetry, i.e., $\eta_{B}>5.75 \times 10^{-10}$. We then calculated the lower bound for successful leptogenesis for different hierarchies $M / M^{\prime}$, and the result is shown in figure 3 . We find that the lowest bound on the scale of leptogenesis is given by

$$
M_{\min }^{\prime} \gtrsim 10^{8} \mathrm{GeV}
$$

obtained when $M / M^{\prime}=3$. Increasing the hierarchy increases the scattering rate for a fixed value of the baryon asymmetry and thus the lower bound increases. Note that the shaded area here denotes the non-hierarchical region $M<3 M^{\prime}$, where our results are not valid. 


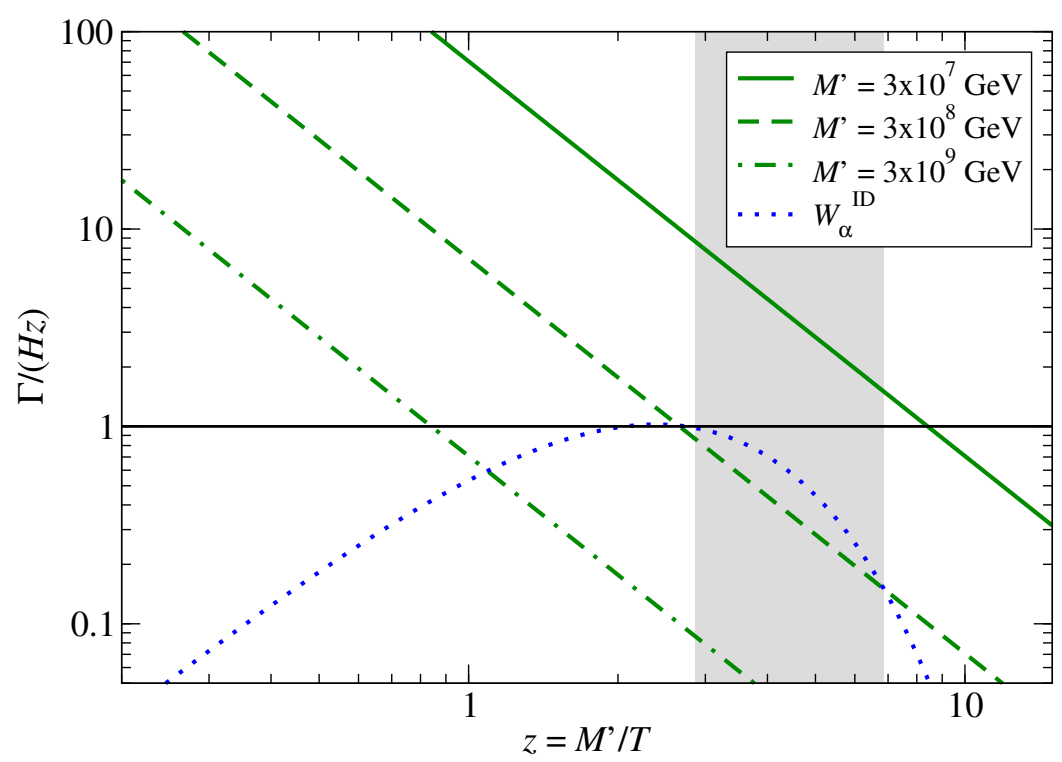

Figure 2. Scattering rate $S_{\alpha \beta}^{\Delta L=0}$ leading to flavour equilibration vs. $z=M^{\prime} / T$ for different values of the lightest heavy neutrino mass $M^{\prime}$. Also plotted is the inverse decay rate $W_{\alpha}^{\mathrm{ID}}$ in flavour $\alpha$, with $K_{1 \alpha}=3.5$. The mass hierarchy has been fixed to $M / M^{\prime}=10$. The shaded region denotes the time at which the asymmetry is produced (see text).

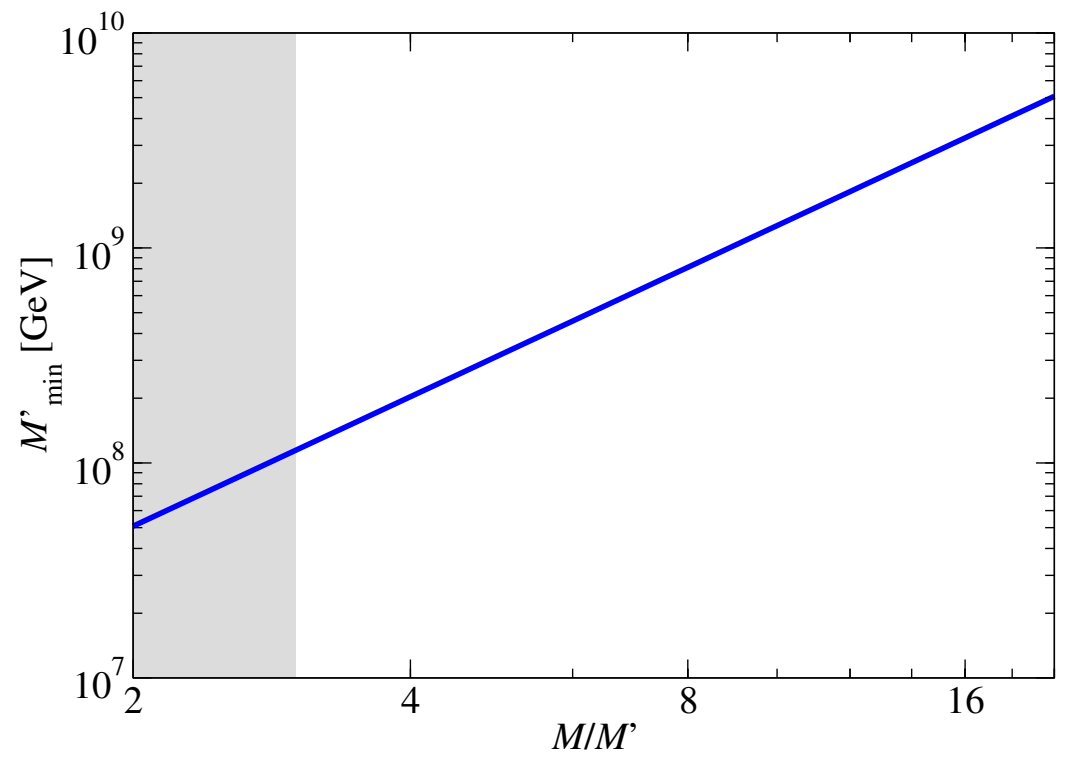

Figure 3. Lower bound on the lightest heavy neutrino mass, $M_{\min }^{\prime}$, for successful leptogenesis, vs. the hierarchy parameter $M / M^{\prime}$. The washout parameter is fixed to $K_{1 \alpha}=3.5$, and the asymmetry is mainly produced in flavour $\alpha$.

It should be noted that this lower bound was obtained maximizing the asymmetry with respect to $K_{1}$ and $K_{1 \alpha}$ and the phases contributing to the $C P$ asymmetry. We have chosen 
$K_{1 \alpha}=3.5$, the smallest value in the strong washout regime [40]. Moreover, we have fixed $K_{1 \beta \neq \alpha}=11$, so that $K_{1}=25.5$, which maximizes the asymmetry. ${ }^{1}$

In our model leptogenesis can take place more than one order of magnitude below the usual lower bound, given by $3 \times 10^{9} \mathrm{GeV}$ at the onset of the strong washout. Unfortunately, it turns out that the coefficient of the $d=6$ operator leading to non-unitarity in eq. (2.8) is expected to be very suppressed, of order $\eta_{\alpha \beta} \sim 10^{-16}$. The reason is mainly the high scale of $M^{\prime}$, and thus $M$, needed for successful leptogenesis. Moreover, the case $M / M^{\prime}=3$ provides the largest non-unitarity possible, since the latter decreases when the scale of $M$ increases. For instance, with $M / M^{\prime}=10$, the Yukawa coupling needed for leptogenesis is $y \simeq 0.07$, in which case we find the lower bound $M_{\text {min }}^{\prime} \simeq 10^{9} \mathrm{GeV}$ and the expected deviation from unitarity is $\eta_{\alpha \beta} \sim 10^{-18}$. We conclude that, in the considered scenario, successful leptogenesis is incompatible with observable non-unitarity signals. Notice that a similar relaxation of the lower bound was obtained in [51] (see also [52]), relying on a $d=7$ operator contribution to the neutrino masses. We did not include this effect since it vanishes in the limit $M_{2}=M_{3}$ and, moreover, has a stronger suppression by the mass hierarchy.

\section{Summary and discussion}

We have investigated the relation between non-unitarity of the leptonic mixing matrix and baryogenesis via thermal leptogenesis. We have first studied how all parameters of the canonical type-I seesaw mechanism can, in principle, be reconstructed from the neutrino mass matrix and a measurement of the deviation of the effective low-energy leptonic mixing matrix from unitary. In the effective low-energy theory, neutrino masses and nonunitarity are encoded in the lepton number violating $d=5$ (Weinberg) operator and in the $d=6$ operator contributing to the neutrino kinetic terms after electroweak symmetry breaking, respectively.

For the case that the mass $M^{\prime}$ of the lightest right-handed neutrino is lighter than the masses of the others, we show that its decay asymmetries for flavour-dependent leptogenesis can be expressed in terms of two contributions, one depending on the unique $d=5$ operator generating neutrino masses and one depending on the $d=6$ operator associated with non-unitarity. We have argued that in low-energy seesaw scenarios, where small lepton number violation explains the smallness of neutrino masses, the lepton number conserving $d=6$ operator contribution, linked to non-unitarity, generically dominates over the $d=5$ operator contribution which results in a strong enhancement of the flavour-dependent decay asymmetries without any resonance effects. We have referred to this case as non-unitarity driven leptogenesis.

To calculate the produced final baryon asymmetry, however, we found that lepton flavour equilibrating effects directly related to non-unitarity play a crucial role and their effects have to be included. In the simple realization of non-unitarity driven leptogenesis considered here, they turn out to forbid lowering the leptogenesis scale down to the

\footnotetext{
${ }^{1}$ If $\left|Y_{1 \beta}\right|$ is too close to $\left|Y_{1 \alpha}\right|$, the washout in all flavours are similar, and the asymmetry is suppressed since $\varepsilon_{1} \simeq 0$. However, if $\left|Y_{1 \beta}\right|$ is much bigger than $\left|Y_{1 \alpha}\right|$, then the $C P$ asymmetry is suppressed [c.f. eq. (4.2)].
} 
TeV scale. Nevertheless, lowering of the leptogenesis scale, i.e., the mass of the lightest right-handed neutrino $M^{\prime}$, to about $10^{8} \mathrm{GeV}$ is possible, which is more than one order of magnitude below the scale of standard thermal leptogenesis. The reduced leptogenesis scale in non-unitarity driven leptogenesis can improve consistency between leptogenesis and gravitino (or similar) constraints in supergravity theories. On the other hand, the deviation from unitarity for the case of $M^{\prime} \gtrsim 10^{8} \mathrm{GeV}$ is far below the experimentally accessible region.

\section{Acknowledgments}

We would like to thank Jacobo Lopez Pavon for useful discussions. S.B. would like to thank the Max-Planck-Institut for Physics for hospitality when this work started. This work was supported by the European Community through the European Commision Marie Curie Actions Framework Programme 7 Intra-European Fellowship: Neutrino Evolution [M.B.]. S.A., M.B. and E.F.M. acknowledge support by the DFG cluster of excellence "Origin and Structure of the Universe". S.A. and E.F.M. also acknowledge support from the European Community under the European Commission Framework Programme 7 Design Study: EUROnu, Project Number 212372.

Open Access. This article is distributed under the terms of the Creative Commons Attribution Noncommercial License which permits any noncommercial use, distribution, and reproduction in any medium, provided the original author(s) and source are credited.

\section{References}

[1] P. Minkowski, $\mu \rightarrow e \gamma$ at a rate of one out of 1-billion muon decays?, Phys. Lett. B 67 (1977) 421 [SPIRES].

[2] R.N. Mohapatra and G. Senjanović, Neutrino mass and spontaneous parity nonconservation, Phys. Rev. Lett. 44 (1980) 912 [SPIRES].

[3] T. Yanagida, Horizontal symmetry and masses of neutrinos, in the proceedings of the Workshop on the Baryon Number of the Universe and Unified Theories, February 13-14, Tsukuba, Japan, (1979).

[4] M. Gell-Mann, P. Ramond and R. Slansky, Complex spinors and unified theories, CERN PRINT-80-0576.

[5] G.C. Branco, W. Grimus and L. Lavoura, The seesaw mechanism in the presence of a conserved lepton number, Nucl. Phys. B 312 (1989) 492 [SPIRES].

[6] A. de Gouvêa, G.F. Giudice, A. Strumia and K. Tobe, Phenomenological implications of neutrinos in extra dimensions, Nucl. Phys. B 623 (2002) 395 [hep-ph/0107156] [SPIRES].

[7] A. Broncano, M.B. Gavela and E.E. Jenkins, The effective Lagrangian for the seesaw model of neutrino mass and leptogenesis, Phys. Lett. B 552 (2003) 177 [hep-ph/0210271] [SPIRES].

[8] M. Fukugita and T. Yanagida, Baryogenesis without grand unification, Phys. Lett. B 174 (1986) 45 [SPIRES].

[9] S. Davidson, E. Nardi and Y. Nir, Leptogenesis, Phys. Rept. 466 (2008) 105 [arXiv:0802.2962] [SPIRES]. 
[10] A. Broncano, M.B. Gavela and E.E. Jenkins, Neutrino physics in the seesaw model, Nucl. Phys. B 672 (2003) 163 [hep-ph/0307058] [SPIRES].

[11] Z.-z. Xing, Casas-Ibarra parametrization and unflavored leptogenesis, arXiv:0902.2469 [SPIRES].

[12] S. Antusch, C. Biggio, E. Fernandez-Martinez, M.B. Gavela and J. Lopez-Pavon, Unitarity of the leptonic mixing matrix, JHEP 10 (2006) 084 [hep-ph/0607020] [SPIRES].

[13] E. Fernandez-Martinez, M.B. Gavela, J. Lopez-Pavon and O. Yasuda, CP-violation from non-unitary leptonic mixing, Phys. Lett. B 649 (2007) 427 [hep-ph/0703098] [SPIRES].

[14] P. Langacker and D. London, Mixing between ordinary and exotic fermions, Phys. Rev. D 38 (1988) 886 [SPIRES].

[15] E. Nardi, E. Roulet and D. Tommasini, Limits on neutrino mixing with new heavy particles, Phys. Lett. B 327 (1994) 319 [hep-ph/9402224] [SPIRES].

[16] D. Tommasini, G. Barenboim, J. Bernabeu and C. Jarlskog, Non-decoupling of heavy neutrinos and lepton flavour violation, Nucl. Phys. B 444 (1995) 451 [hep-ph/9503228] [SPIRES].

[17] S. Antusch, J.P. Baumann and E. Fernandez-Martinez, Non-standard neutrino interactions with matter from physics beyond the standard model, Nucl. Phys. B $\mathbf{8 1 0}$ (2009) 369 [arXiv:0807.1003] [SPIRES].

[18] J.A. Casas and A. Ibarra, Oscillating neutrinos and $\mu \rightarrow e, \gamma$, Nucl. Phys. B 618 (2001) 171 [hep-ph/0103065] [SPIRES].

[19] A. Abada, C. Biggio, F. Bonnet, M.B. Gavela and T. Hambye, Low energy effects of neutrino masses, JHEP 12 (2007) 061 [arXiv:0707.4058] [SPIRES].

[20] B. Bekman, J. Gluza, J. Holeczek, J. Syska and M. Zralek, Matter effects and CP-violating neutrino oscillations with non-decoupling heavy neutrinos, Phys. Rev. D 66 (2002) 093004 [hep-ph/0207015] [SPIRES].

[21] J. Holeczek, J. Kisiel, J. Syska and M. Zralek, Searching for new physics in future neutrino factory experiments, Eur. Phys. J. C 52 (2007) 905 [arXiv:0706.1442] [SPIRES].

[22] S. Goswami and T. Ota, Testing non-unitarity of neutrino mixing matrices at neutrino factories, Phys. Rev. D 78 (2008) 033012 [arXiv:0802.1434] [SPIRES].

[23] G. Altarelli and D. Meloni, CP violation in neutrino oscillations and new physics, Nucl. Phys. B 809 (2009) 158 [arXiv: 0809.1041] [SPIRES].

[24] S. Antusch, M. Blennow, E. Fernandez-Martinez and J. Lopez-Pavon, Probing non-unitary mixing and CP-violation at a neutrino factory, Phys. Rev. D 80 (2009) 033002 [arXiv: 0903.3986] [SPIRES].

[25] M.B. Gavela, T. Hambye, D. Hernandez and P. Hernández, Minimal flavour seesaw models, JHEP 09 (2009) 038 [arXiv:0906.1461] [SPIRES].

[26] L. Covi, E. Roulet and F. Vissani, CP violating decays in leptogenesis scenarios, Phys. Lett. B 384 (1996) 169 [hep-ph/9605319] [SPIRES].

[27] A. Pilaftsis, CP violation and baryogenesis due to heavy Majorana neutrinos, Phys. Rev. D 56 (1997) 5431 [hep-ph/9707235] [SPIRES].

[28] M.C. Gonzalez-Garcia, J. Racker and N. Rius, Leptogenesis without violation of B-L, JHEP 11 (2009) 079 [arXiv:0909.3518] [SPIRES]. 
[29] A. Abada, S. Davidson, F.-X. Josse-Michaux, M. Losada and A. Riotto, Flavour issues in leptogenesis, JCAP 04 (2006) 004 [hep-ph/0601083] [SPIRES].

[30] E. Nardi, Y. Nir, E. Roulet and J. Racker, The importance of flavor in leptogenesis, JHEP 01 (2006) 164 [hep-ph/0601084] [SPIRES].

[31] D. Aristizabal Sierra, L.A. Muñoz and E. Nardi, Purely flavored leptogenesis, Phys. Rev. D 80 (2009) 016007 [arXiv:0904.3043] [SPIRES].

[32] W. Buchmüller, P. Di Bari and M. Plümacher, Leptogenesis for pedestrians, Ann. Phys. 315 (2005) 305 [hep-ph/0401240] [SPIRES].

[33] S. Blanchet and P. Di Bari, New aspects of leptogenesis bounds, Nucl. Phys. B 807 (2009) 155 [arXiv:0807.0743] [SPIRES].

[34] S.Y. Khlebnikov and M.E. Shaposhnikov, The statistical theory of anomalous fermion number nonconservation, Nucl. Phys. B 308 (1988) 885 [SPIRES].

[35] J.A. Harvey and M.S. Turner, Cosmological baryon and lepton number in the presence of electroweak fermion number violation, Phys. Rev. D 42 (1990) 3344 [SPIRES].

[36] G.F. Giudice, A. Notari, M. Raidal, A. Riotto and A. Strumia, Towards a complete theory of thermal leptogenesis in the SM and MSSM, Nucl. Phys. B 685 (2004) 89 [hep-ph/0310123] [SPIRES].

[37] WMAP collaboration, E. Komatsu et al., Five-year Wilkinson Microwave Anisotropy Probe (WMAP) observations: cosmological interpretation, Astrophys. J. Suppl. 180 (2009) 330 [arXiv: 0803.0547] [SPIRES].

[38] S. Davidson and A. Ibarra, A lower bound on the right-handed neutrino mass from leptogenesis, Phys. Lett. B 535 (2002) 25 [hep-ph/0202239] [SPIRES].

[39] W. Buchmüller, P. Di Bari and M. Plümacher, Cosmic microwave background, matter-antimatter asymmetry and neutrino masses, Nucl. Phys. B 643 (2002) 367 [hep-ph/0205349] [SPIRES].

[40] S. Blanchet and P. Di Bari, Flavor effects on leptogenesis predictions, JCAP 03 (2007) 018 [hep-ph/0607330] [SPIRES].

[41] S. Antusch and A.M. Teixeira, Towards constraints on the SUSY seesaw from flavour-dependent leptogenesis, JCAP 02 (2007) 024 [hep-ph/0611232] [SPIRES].

[42] M.Y. Khlopov and A.D. Linde, Is it easy to save the gravitino?, Phys. Lett. B 138 (1984) 265 [SPIRES].

[43] J.R. Ellis, D.V. Nanopoulos, K.A. Olive and S.-J. Rey, On the thermal regeneration rate for light gravitinos in the early universe, Astropart. Phys. 4 (1996) 371 [hep-ph/9505438] [SPIRES].

[44] T. Moroi, H. Murayama and M. Yamaguchi, Cosmological constraints on the light stable gravitino, Phys. Lett. B 303 (1993) 289 [SPIRES].

[45] J. Pradler and F.D. Steffen, Constraints on the reheating temperature in gravitino dark matter scenarios, Phys. Lett. B 648 (2007) 224 [hep-ph/0612291] [SPIRES].

[46] D. Aristizabal Sierra, M. Losada and E. Nardi, Lepton flavor equilibration and leptogenesis, JCAP 12 (2009) 015 [arXiv: 0905.0662] [SPIRES].

[47] A. Pilaftsis and T.E.J. Underwood, Electroweak-scale resonant leptogenesis, Phys. Rev. D 72 (2005) 113001 [hep-ph/0506107] [SPIRES]. 
[48] W. Buchmüller and M. Plümacher, Spectator processes and baryogenesis, Phys. Lett. B 511 (2001) 74 [hep-ph/0104189] [SPIRES].

[49] E. Nardi, Y. Nir, J. Racker and E. Roulet, On Higgs and sphaleron effects during the leptogenesis era, JHEP 01 (2006) 068 [hep-ph/0512052] [SPIRES].

[50] S. Blanchet and P. Di Bari, Leptogenesis beyond the limit of hierarchical heavy neutrino masses, JCAP 06 (2006) 023 [hep-ph/0603107] [SPIRES].

[51] M. Raidal, A. Strumia and K. Turzynski, Low-scale standard supersymmetric leptogenesis, Phys. Lett. B 609 (2005) 351 [hep-ph/0408015] [SPIRES].

[52] T. Hambye, Y. Lin, A. Notari, M. Papucci and A. Strumia, Constraints on neutrino masses from leptogenesis models, Nucl. Phys. B 695 (2004) 169 [hep-ph/0312203] [SPIRES]. 